\title{
Feedback in the Dark Domain
}

\author{
Keiichi Wada $\mathbf{a}^{1,2}$ \\ ${ }^{1}$ National Astronomical Observatory of Japan \\ Email: wada@cfca.jp \\ ${ }^{2}$ Graduate School of Science and Engineering, Kagoshima University, \\ Kagoshima 890-0065, Japan
}

\begin{abstract}
In order to clarify the physics of AGN feedback and feeding, we need to understand the interstellar medium (ISM) in the central several tens of parsecs in galaxies where our observational and theoretical knowledge is relatively poor. Here we discuss feedback processes due to strong far UV and X-ray radiation, as well as the dynamical effect of a supermassive black hole $(\mathrm{SMBH})$ based on new high-resolution numerical simulations of the ISM in the central $R \leqslant 32$ $\mathrm{pc}$ region around a $\mathrm{SMBH}$ at the center of a galaxy.
\end{abstract}

Keywords. methods: numerical, galaxies: evolution, galaxies: ISM

\section{What Can We See in the Dark?}

The dynamic range that is pertinent to feeding and feedback is enormous, from scales of $\mathrm{AU}$ (the accretion disk) to megaparsecs (clusters of galaxies). Not all domains have been observationally resolved. We have some multiwavelength spectroscopic information on the accretion disks, ionized gas regions, and hot dust, all of which are few parsecs or much smaller in size, in the X-ray, optical, UV, and infrared. Yet the spatial structure of the AGNs themselves is still unclear even in nearby galaxies. On the other hand, the structure of the ISM outside the central core $(r>100 \mathrm{pc})$ in nearby galaxies is resolved by multiwavelength observations. There is a big unresolved "gap" between these two regions. I therefore call this central region within several tens of parsecs of the black hole "the dark domain." In many spiral galaxies, concentrations of molecular gas are observed at their centers (Kohno et al. 2003). The dark domain is inside this molecular core.

Recently we have some obtained some information on the structure and kinematics of hot molecular gas in the central tens of parsecs in nearby galaxies by using the VLT and Keck (Hicks et al. 2009 and these proceedings), which shows that it is an inhomogeneous and turbulent region. This is important progress, but we still need ALMA to clarify the structure of the cold molecular gas in the dark domain.

Fabian (these proceedings) has emphasized that AGNs have strong dynamical effects on galactic or larger scales, but potentially AGNs could also affect their environment on all scales between the central $\mathrm{BH}$ and clusters of galaxies. In this paper, I would like to focus on the structure of the ISM in the dark domain and discuss feedback processes from a theoretical point of view. We should consider the "sign" and "effectiveness" of the feedback processes. If it is negative in terms of mass accretion to the central parsec and it is very efficient, then we do not expect steady AGN activity because feedback will prevent fueling. On the other hand, if the feedback works positively and its efficiency is high, AGN activity becomes a runaway process and it could blow out the ISM by radiation pressure and radiative heating, and again the AGN cannot be steady. Yet we observe many AGNs, which suggests that the feedback should be moderate regardless of its sign at least during the AGN lifetime, or there should be some mechanism to regulate the feedback. 
As discussed elsewhere in these proceedings, there are in fact many kinds of feedback processes. Radiative feedback is discussed by Proga and by Fabian in these proceedings and also in a series of papers by Ohsuga and collaborators (e.g., Ohsuga et al. 2009). In the dark domain, chemical feedback is also important, so I will briefly mention feedback due to X-ray radiation from the AGNs based on models of X-ray dominated regions (XDRs). What I would like to emphasize here is that in order to understand all these processes, it is crucial to understand at least the three-dimensional structure of the ISM.

In the simplest uniform model of the obscuring torus, silicate features are predicted to be in emission in type 1 AGNs and in absorption in type 2 AGNs. Shi et al. (2006) show the strength of the $9.7 \mathrm{~mm}$ silicate feature in various AGNs as a function of the column density toward the nucleus. It is clear that there is a trend of more absorption in AGNs with larger column density, but the scatter is large, and there are many exceptions. A simple explanation of this is that the obscuring material is distributed in a highly inhomogeneous manner, and the traditional uniform "doughnut-like" torus model should be rejected. SED model fitting also supports a clumpy torus model (Schartmann et al. 2008).

Wada \& Norman (2002; hereafter WN02) modeled the molecular "torus" using fully time-dependent three-dimensional hydrodynamic calculations. They investigated evolution of the ISM in the inner $100 \mathrm{pc}$ around a $10^{8} M_{\odot} \mathrm{SMBH}$ using three-dimensional Euler-grid hydrodynamic simulations, taking into account self-gravity of the gas, radiative cooling, and heating due to supernovae. They found that a thick disk whose size is several tens of parsecs is naturally reproduced around a SMBH. The internal density and temperature are highly inhomogeneous and the velocity field is turbulent, but the global geometry, i.e., a flared thick disk, is quasi-stable, implying that the energy input from supernovae is balanced by the turbulent dissipation. They suggest that AGNs could be obscured by the surrounding interstellar material. These results are partially supported by that observational findings that some AGNs are obscured by nuclear starbursts (Levenson et al. 2007; Ballantyne 2008). However, the models of WN02 are not necessarily applicable to all types of AGNs with active star formation, because (1) the assumed supernova rate is extremely high $\left(\sim 10^{-4} \mathrm{yr}^{-1} \mathrm{pc}^{-2}\right)$, which would be appropriate only for intense starbursts, like ultraluminous infrared galaxies, but not to standard circumnuclear starbursts (Hicks et al. 2009), and (2) the cold, dense gas in their simulations does not necessarily represent the dusty molecular gas phase around an AGN, because $\mathrm{H}_{2}$ chemistry is not included and therefore the radiative cooling rate used by WN02 below several hundred Kelvin is not self-consistent.

We introduce here our new three-dimensional hydrodynamic simulations that extend the work of WN02 to explicitly track the evolution of the $\mathrm{H}_{2}$ phase, and its interplay with the $\mathrm{H}$ I phase with $0.125 \mathrm{pc}$ resolution (compared to $0.25 \mathrm{pc}$ by WN02) in the central tens of parsecs. We also vary the strength of the uniform FUV field and the supernova rate (SNR) in order to examine its effect on the structure of molecular gas clouds (see Wada et al. 2009 for details).

\section{Structure of Multi-Phase Gas Around a SMBH}

\subsection{Methods and Models}

We use a numerical scheme based on Eulerian hydrodynamics with a uniform grid by which mass, momentum, and energy equations are solved with the Poisson equation to simulate the three-dimensional evolution of a rotating gas disk in a fixed spherical gravitational potential. The hydrodynamic code is called HD. ${ }^{*}$, in which the hydrodynamic 
part is solved by the advection upstream splitting method (AUSM) as described by Wada $\&$ Norman (2001). We use $512^{2} \times 256$ grid points in high-resolution models and $256^{2} \times 128$ grid points in low-resolution models to investigate long-term evolution with different parameters. Cartesian grid points cover a $64 \times 64 \times 32 \mathrm{pc}^{3}$ region around the galactic center (i.e., the spatial resolution is $0.125 \mathrm{pc}$ in the high-resolution runs and $0.25 \mathrm{pc}$ for the low resolution runs). To calculate the self-gravity of the gas, we employ a Fast Fourier Transform and the convolution method, where $1024^{2} \times 512$ grid points and a periodic Green's function are used.

An essential improvement in the present calculations compared to those of WN02 is that we now solve the non-equilibrium chemistry of hydrogen molecules along with the hydrodynamics. Formation of $\mathrm{H}_{2}$ on dust and its radiative and collisional destruction are explicitly tracked, so we can deduce the distribution of $\mathrm{H}_{2}$ in the central tens of parsecs.

We use a cooling function based on a radiative transfer model of photo-dissociation regions (Meijerink \& Spaans 2005), with $\Lambda\left(T_{g}, f_{\mathrm{H}_{2}}, G_{0}\right)$ over the range $\left(20 \mathrm{~K} \leqslant T_{g} \leqslant\right.$ $10^{8} \mathrm{~K}$ ) assuming solar metallicity, which is a function of the molecular gas fraction $f_{\mathrm{H}_{2}}$ and intensity of FUV radiation $G_{0}$. We assume a uniform UV radiation field, whose strength is an important parameter in the model, that is represented by the Habing unit $\left(G_{0}\right)$. The radiative cooling rate below $\sim 10^{4} \mathrm{~K}$ is self-consistently modified depending on $f_{\mathrm{H}_{2}}$, which is determined in each grid cell. We assume heating $\Gamma_{\mathrm{UV}}$ due to the photoelectric effect and energy feedback $\Gamma_{\mathrm{SN}}$ from type II SNe.

The initial condition is an axisymmetric and rotationally supported thin disk with a uniform density profile with a thickness of $2.5 \mathrm{pc}$ and a total gas mass of $M_{g}=6 \times 10^{6} M_{\odot}$. Since we allow outflows from the computational boundaries, the total gas mass decreases during the evolution, and settles to $\sim 5 \times 10^{6} M_{\odot}$ at a quasi-equilibrium state. The system settles into a quasi-equilibrium state where the total molecular gas mass is nearly constant after $t \sim 3.5$ Myr. We assume a spatially uniform FUV field with $G_{0}=10,100$, and 1000 . In reality, both the density field and distribution of massive stars are not uniform, and $G_{0}$ should have a large dispersion with some radial dependence. The effect of a non-uniform radiation field will be an important subject to address with three-dimensional radiative transfer calculations in the future.

\subsection{Inhomogeneous Torus}

In a quasi-steady state $(t \gtrsim 3.5 \mathrm{Myr})$, as reported by WN02, the gas forms a highly inhomogeneous flared disk, as seen in Figure 1, which shows gas density, temperature, and molecular hydrogen density at $t=4.38 \mathrm{Myr}$. The temperature map shows that cold $\left(T_{g} \lesssim 100 \mathrm{~K}\right)$ gas is mainly distributed in the high-density regions. In the central funnellike cavity, the temperature of the gas is hot $\left(T_{g} \gtrsim 10^{6} \mathrm{~K}\right)$. Hot gas is also patchily distributed in the cold, flared disk. The typical size of these hot cavities is a few parsecs. A large fraction of the volume is occupied by warm gas $\left(T_{g} \sim 8000 \mathrm{~K}\right)$. In Figure 2 , the distribution of $\mathrm{H}_{2}$ is shown; it roughly follows the cold, dense gas, and therefore forms a high-density circumnuclear disk whose radius is about $5 \mathrm{pc}$, surrounded by a porous torus which extends to $\sim 5-10 \mathrm{pc}$ above the disk plane.

The torus shows global rotation, but there are also large internal random motions. This complicated velocity field is naturally expected in an inhomogeneous, self-gravitating disk (Wada, Meurer, \& Norman 2002), but the vertical motions are mainly enhanced by energy input from supernovae. The vertical velocity field also shows a bipolar outflow in the central funnel, which is mostly warm and hot gases as seen in Figure 1.

In Figure 3, we plot total column density $\left(N_{\mathrm{g}}\right)$ toward the galactic center as a function of the viewing angle for the same model shown in Figure 1. It clearly shows that the total gas and $\mathrm{H}_{2}$ column densities are both largest at $\theta_{v} \sim 0$ (i.e., edge-on), and the 
average column density, shown by the thin solid line, decreases toward the pole-on view, as expected. We should note, however, that the scatter around the average is two orders of magnitude or more for any viewing angle, which is comparable to the change of the average value between $\theta_{v}=0^{\circ}$ and $\pm 90^{\circ}$.

\section{Various Feedback Processes}

\subsection{The Effect of FUV Radiation}

In Figure 4, evolution of the $\mathrm{H}_{2}$ molecular gas fraction, $f_{\mathrm{H}_{2}} \equiv M_{\mathrm{H}_{2}} / M_{\text {total }}$ is shown for models with $G_{0}=10$ (model H10a), 100 (model H100a), and 1000 (model H1000a). The $\mathrm{H}_{2}$ molecular gas fraction in H10a rapidly decreases from $f_{\mathrm{H}_{2}} \sim 0.5$ to $\sim 0.32$ in the first Myr, and then increases and slightly oscillates around $f_{\mathrm{H}_{2}} \sim 0.4$. The models with ten and one hundred times stronger FUV (H100a and H1000a) show similar time evolution. The difference is small, and even for $G_{0}=1000, f_{\mathrm{H}_{2}} \sim 0.3$ at $t \sim 4 \mathrm{Myr}$. This weak dependence on $G_{0}$ is caused by the $\mathrm{H}_{2}$ self-shielding against FUV dissociation in highdensity cores. This suggests that the FUV feedback on the amount of $\mathrm{H}_{2}$ is negative, but weak, therefore we expect that star formation is regulated in the dark domain.

\subsection{Effects of $X$-Rays}

The strong X-ray continuum emanating from the AGN could affect the chemical state of the ISM in the central region (Maloney et al. 1996; Meijerink et al. 2007). It is known that the chemical state of XDRs is mainly determined by $H_{\mathrm{X}} / n$, where $H_{\mathrm{X}}$ is a X-ray heating deposition rate and $n$ is the number density of the gas (Maloney et al. 1996). Although we do not explicitly include the effect of X-rays from the nucleus in the hydrodynamical simulations, here we estimate its potential effect from one of our results. In Figure 5, we
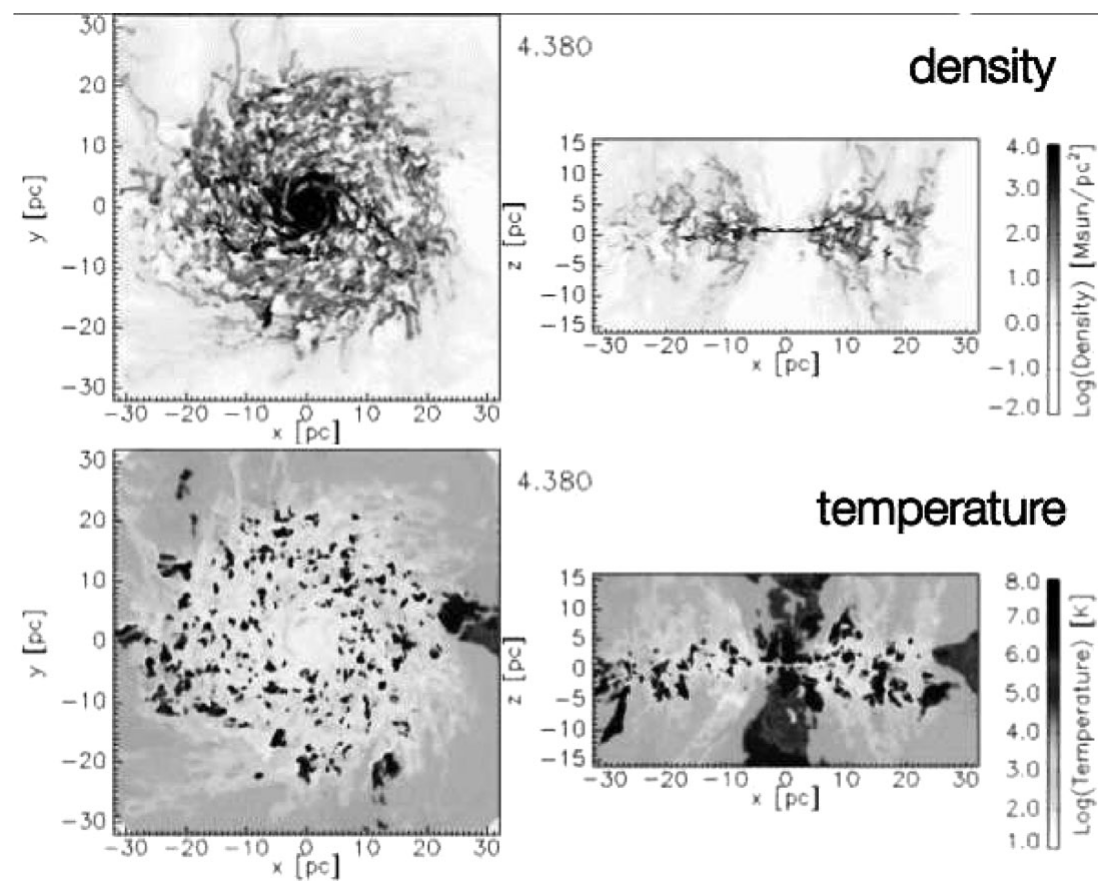

Figure 1. Density (top) and temperature (bottom) distributions of a model with $G_{0}=10$ in a quasi-steady state. 
plot the fraction of $\mathrm{H}_{2}$ and temperature as a function of $H_{\mathrm{X}} / n\left(\mathrm{ergs} \mathrm{cm}^{3} \mathrm{~s}^{-1}\right)$ at randomly selected points in the computational box. Here we assume that the X-ray luminosity of the AGN is $L_{\mathrm{X}}=10^{44} \mathrm{ergs} \mathrm{s}^{-1}$. Although there is large scatter, for $\log \left(H_{\mathrm{X}} / n\right) \sim-22$ or smaller, the molecular fraction increases significantly and the temperature in most regions is less than $100 \mathrm{~K}$. At high gas densities $\left(\sim 10^{5} \mathrm{~cm}^{-3}\right)$, large $(>0.1) \mathrm{H}_{2}$ fractions can be sustained for the range of $H_{\mathrm{X}} / n$ values, limiting the impact of XDR effects (Meijerink et al. 2007). This is partly because the free electrons associated with ionization processes help to form $\mathrm{H}_{2}$ through the $\mathrm{H}^{-}$route and also because the $\mathrm{H}_{2}$ formation scales with density squared. Still, for $H_{\mathrm{X}} / n$ significantly larger than $10^{-26}$, gas is mostly atomic as expected (Maloney et al. 1996). In Figure 5, we also show a rough estimate of the $\mathrm{H}_{2}$ fraction as a function of $H_{\mathrm{X}} / n$ for $L_{\mathrm{X}}=10^{42}$ and $10^{44} \mathrm{ergs} \mathrm{s}^{-1}$, based on calculations by Maloney et al. (1996). This suggests that high-density clouds with $f_{\mathrm{H}_{2}}>0.1$ could be affected by an X-ray source with $L_{\mathrm{X}}=10^{42} \operatorname{ergs~s}^{-1}$.

The $\mathrm{H}_{2}$ abundance is indeed robust in a clumpy medium, but the XDR gas temperature should rise relative to the PDR case by a factor of $\sim 5$ for $\log H_{\mathrm{X}} / n>-26$ (Meijerink et al. 2007). After all, X-ray ionization heating with, for example, $H_{\mathrm{X}} / n \sim 10^{-25} \mathrm{ergs} \mathrm{s}^{-1}$, is more efficient than photoelectric emission by dust grains, and therefore $\mathrm{H}_{2}$ gas would have higher temperatures. This leads to stronger emission in the pure rotational $\mathrm{H}_{2}$ lines, such as $S(0)$ and $S(1)$ at $28 \mu \mathrm{m}$ and $17 \mu \mathrm{m}$, respectively.

\subsection{Gravitational Feedback}

The black hole itself could dynamically affect the mass accretion process through stability of the circumnuclear disk in the central few tens pf parsecs. For a given gas density in the disk, if the BH mass is small enough, the whole disk can be fully unstable, then stars could be formed. As a result, turbulent motion of the gas is generated by energy feedback from stars. This makes the disk thick and enhances the kinematic viscosity. When the $\mathrm{BH}$ grows, the inner part of the disk becomes gravitationally stable and thin, supported by thermal pressure. In this phase, the mass accretion rate is in general smaller than that in the turbulence-dominated unstable disk. One of important results predicted by this picture is that the "efficiency" of BH growth in terms of mass supply from a host galaxy is much smaller than $100 \%$, especially for more massive BHs. (Kawakatu \& Wada 2008, 2009). Figure 6 shows the final BH mass as a function of total accreted mass from the host galaxy. The solid line is our prediction. The dot-dashed line denotes $M_{\mathrm{BH} \text {,final }}=M_{\mathrm{sup}}$ (i.e., $100 \%$ efficiency). It shows that the final BH mass is much smaller than $M_{\text {sup }}$. This means that, even if a large amount of the gas is supplied to the circumnuclear region, a large fraction of the gas is consumed by star formation and not used to grow the BH. In

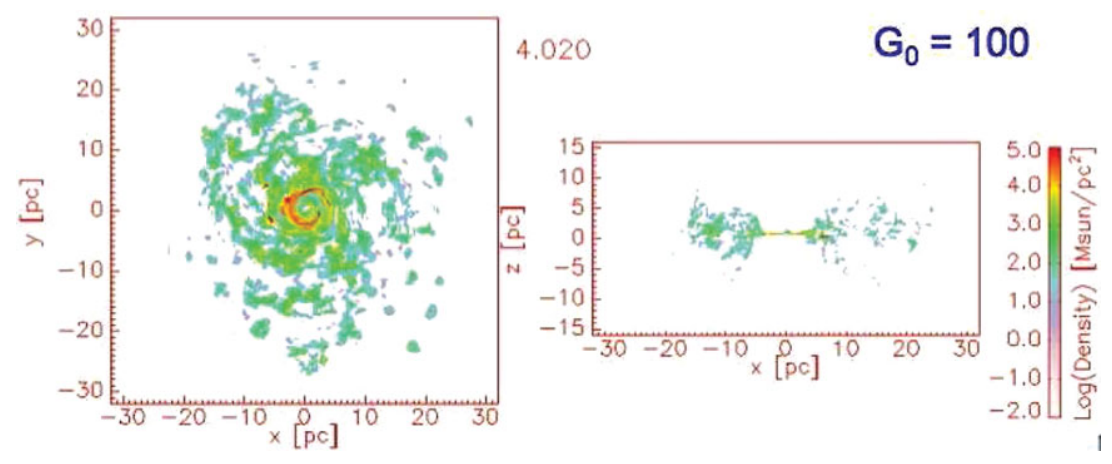

Figure 2. Molecular hydrogen distribution in a model with $G_{0}=100$. 
this sense, growth of the BH itself is negative feedback. This is an especially important consideration for the origin of high- $z$ QSOs (Kawakatu \& Wada 2009).

\section{Summary}

1. The ISM in the central several tens of parsecs in galaxies is a key to understanding the evolution of AGNs and co-evolution of supermassive black holes (SMBHs) and host galaxies, but observational information is still insufficient in this "dark domain."

2. Since the feedback and feeding processes in the dark domain should depend on the physical state of the ISM, it is important to understand the three-dimensional multiphase structure of the ISM.

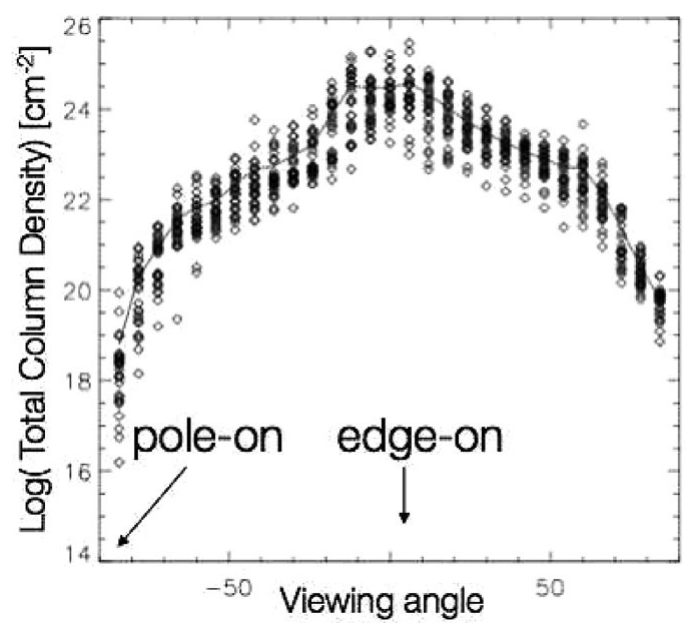

Figure 3. Column density toward the nucleus as a function of the viewing angle.

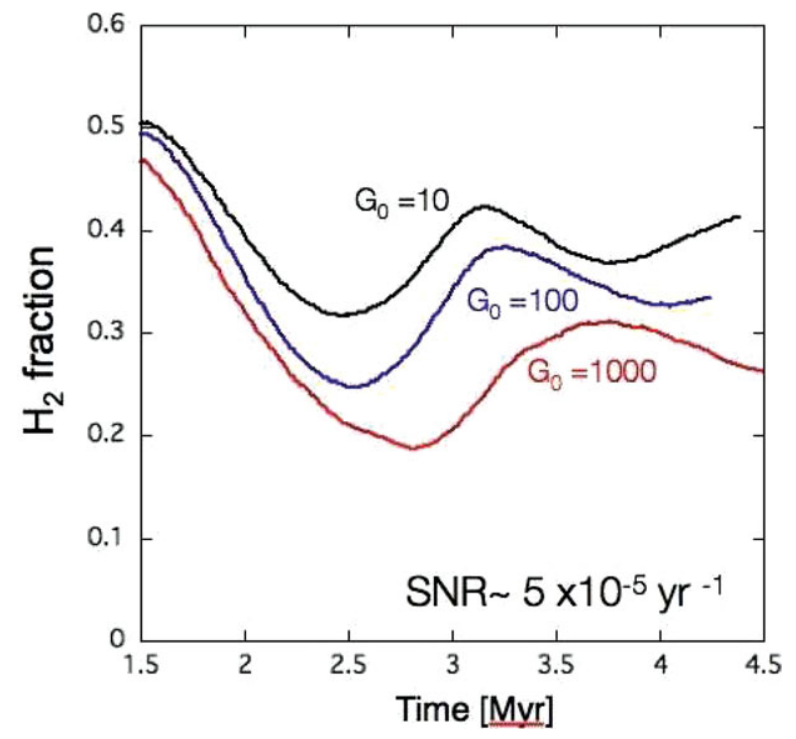

Figure 4. $\mathrm{H}_{2}$ fraction as a function of FUV. 


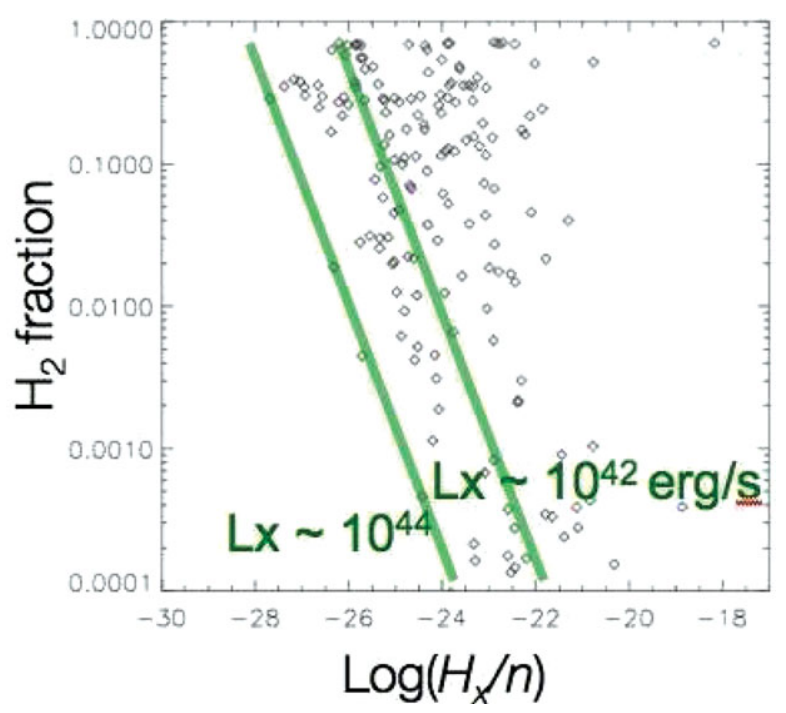

Figure 5. A fraction of $\mathrm{H}_{2}$ is plotted as a function of $H_{\mathrm{X}} / n\left(\mathrm{ergs} \mathrm{cm}^{3} \mathrm{~s}^{-1}\right)$ at randomly selected points in the computational box. The X-ray luminosity of the AGN is $L_{\mathrm{X}}=10^{44} \mathrm{ergs} \mathrm{s}^{-1}$. Two solid lines represent predictions from the XDR modeling (Maloney et al. 1996) for $L_{\mathrm{X}}=10^{42} \operatorname{ergs~s}^{-1}$ and $L_{\mathrm{X}}=10^{42} \mathrm{ergs} \mathrm{s}^{-1}$.

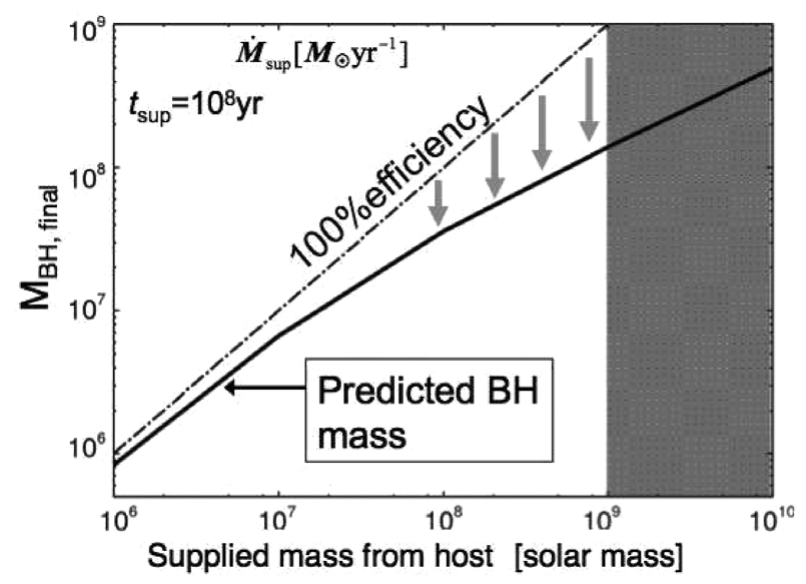

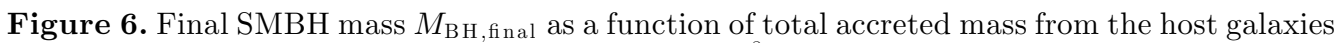
$M_{\text {sup }}$. The time scale of the mass supply is $t_{\text {sup }}=10^{8} \mathrm{yr}$; " $100 \%$ efficiency" means that all the mass supplied is used to grow the SMBH, i.e., $M_{\mathrm{BH}, \mathrm{final}}=M_{\mathrm{sup}}$, although this is not the case in our theoretical model (for details see Kawakatu \& Wada 2009).

3. We performed new high-resolution numerical simulations of the ISM in a central $R \leqslant 32$-parsec region around a supermassive black hole at a galactic center. Threedimensional hydrodynamic modeling of the ISM (Wada \& Norman 2002) with a nuclear starburst now includes tracking the formation of molecular hydrogen out of the neutral hydrogen phase as a function of the evolving ambient ISM conditions with finer spatial resolution $(0.125 \mathrm{pc})$. We find that

- A small supernova rate $\left(\sim 10^{-5} \mathrm{yr}^{-1}\right)$ is large enough to enhance turbulent motions in the inhomogeneous disk, and as a result the disk becomes geometrically thick. 
- Total and $\mathrm{H}_{2}$ column densities toward the nucleus are not a single function of position vectors. For a given viewing angle, scatter as large as one or two orders of magnitude is found due to the inhomogeneous density field of the gas.

- Molecular hydrogen in the clumpy media is destroyed by strong FUV radiation, but the total mass of $\mathrm{H}_{2}$ decreases by $20-30 \%$ even if the FUV is $10-100$ times stronger. In this sense, the feedback effect of massive star formation is negative, but weak.

- X-rays from the AGN could have a strong negative feedback effect on $M_{\mathrm{H} 2}$.

- Growth of the SMBH itself provides negative feedback, i.e., the growth in more massive SMBH is less efficient because the mass accretion becomes smaller due to to kinematic viscosity in the turbulent, clumpy "torus."

\section{References}

Ballantyne, D. R. 2008, ApJ, 685, 787

Hicks, E. K. S., Davies, R. I., Malkan, M. A., Genzel, R., Tacconi, L. J., Sánchez, F. M., \& Sternberg, A. 2009, ApJ, 696, 448

Kawakatu, N. \& Wada, K. 2008, ApJ, 681, 73

Kawakatu, N. \& Wada, K. 2009, ApJ, 706, 676

Kohno, K., Ishizuki, S., Matsushita, S., Vila-Vilaró, B., \& Kawabe, R. 2003, PASJ, 55, L1

Levenson, N. A., Sirocky, M. M., Hao, L., Spoon, H. W. W., Marshall, J. A., Elitzur, M., \& Houck, J. R. 2007, ApJ, 654, L45

Maloney, P. R., Hollenbach, D. J., \& Tielens, A. G. G. M. 1996, ApJ, 466, 561

Meijerink, R. \& Spaans, M. 2005, A\&A, 436, 397

Meijerink, R., Spaans, M., \& Israel, F. P. 2007, A\& $A$, 461, 793

Ohsuga, K., Mineshige, S., Mori, M., \& Kato, Y. 2009, PASJ, 61, L7

Schartmann, M., Meisenheimer, K., Klahr, H., Camenzind, M., Wolf, S., \& Henning, T. 2009, MNRAS, 393, 759

Schartmann, M., Meisenheimer, K., Camenzind, M., Wolf, S., Tristram, K. R. W., \& Henning, T. 2008, $A \& A, 482,67$

Shi, Y., et al. 2006, ApJ, 653, 127

Spaans, M. \& Meijerink, R. 2008, ApJ, 678, L5

Wada, K. \& Norman, C. A. 2001, ApJ, 547, 172

Wada, K. \& Norman, C. A. 2002, ApJ, 566, L21 (WN02)

Wada, K., Meurer, G., \& Norman, C. A. 2002, ApJ, 577, 197

Wada, K., Papadopoulos, P., \& Spaans, M. 2009, ApJ, 702, 63 